\title{
Propofol General Anesthesia Decreases the Coupling Strength Between Mean Arterial Blood Pressure and Mean Cerebral Blood Flow Velocity in Patients Undergoing Coronary Artery Bypass Grafting
}

\author{
Vlasta Bari ${ }^{1}$, Emanuele Vaini ${ }^{1}$, Angela Fantinato ${ }^{1}$, Beatrice De Maria ${ }^{2}$, Beatrice Cairo ${ }^{3}$, \\ Valeria Pistuddi ${ }^{1}$, Marco Ranucci ${ }^{1}$, Alberto Porta ${ }^{1,3}$ \\ ${ }^{1}$ Department of Cardiothoracic, Vascular Anesthesia and Intensive Care, IRCCS Policlinico San \\ Donato, San Donato Milanese, Milan, Italy \\ ${ }^{2}$ IRCCS Istituti Clinici Scientifici Maugeri, Milan, Italy \\ ${ }^{3}$ Department of Biomedical Sciences for Health, University of Milan, Milan, Italy
}

\begin{abstract}
Cerebral autoregulation (CA) maintains mean cerebral blood flow velocity (MCBFV) stable in spite of variations in mean arterial pressure (MAP). Propofol anesthesia is known to preserve $C A$, although information on the anesthesia effect on MCBFV and MAP variability coupling at sedation levels typical of major cardiac surgery is limited. MAP and MCBFV time series were respectively recorded from radial artery and left middle cerebral artery via transcranial Doppler, before (PRE) and after (POST) general anesthesia induction with propofol and remifentanil in 10 male subjects (age $64.7 \pm 7.0$ years) undergoing coronary artery bypass grafting. Squared coherence $\left(K^{2}\right)$ was computed in the typical bands of CA, namely very low (0.02-0.07 Hz), low (0.07-0.15 Hz) and high frequency $(0.15-0.4 \mathrm{~Hz})$. The null hypothesis of coupling was tested through a surrogate analysis associating MAP and MCBFV series taken from different patients in the same experimental condition. $M A P$ and MCBFV original series were significantly more associated than surrogate ones during PRE. $K^{2}$ was reduced in the three frequency bands during POST to a level comparable with surrogates. By decoupling $M C B F V$ and MAP propofol general anesthesia favors situations of stable MCBFV in response to slow modifications of MAP but the full decoupling might hide the limited resources of the cerebrovascular control to actively regulate $M C B F V$.
\end{abstract}

\section{Introduction}

Cerebral autoregulation (CA) is a regulatory mechanism aiming at maintaining a relatively constant cerebral blood flow $(\mathrm{CBF})$ in presence of slow changes in arterial pressure (AP) [1]. An intervention evoking a transient variation of mean AP (MAP) is applied to probe the CA functioning (e.g. the transient hypotension induced by thigh cuff rapid deflation) [2]. However, in settings in which MAP cannot be modified for ethical reason (e.g. during general anesthesia or critical care unit), CA is probed using spontaneous variability of MAP and mean CBFV (MCBFV) [1]. CA is known to have a fundamental role in maintaining cerebral perfusion in presence of challenging situations such as during standing [3], it is influenced by sympathetic vascular regulation [4], and it is altered in pathology $[5,6]$.

During major cardiac surgery a working CA might be advisable because the organism would be able to react to situations of hypertension or hypoperfusion with suitable adjustments of vascular resistances. Previous works suggest that volatile anesthesia impairs CA, while propofol anesthesia maintains it $[7,8]$. However, some modifications are expected given that $\mathrm{CA}$ is partially mediated by autonomic nervous system [4] and propofol general anesthesia is well-known depressor of autonomic and vascular regulation [9]. Moreover, previous studies assessed the effect of propofol at doses lower than those applied during major cardiac surgery [8] or using interventional approaches to probe CA [7], thus limiting our knowledge about the effect of propofol anesthesia on $\mathrm{CA}$, especially in critical settings where deep sedation is necessary and interventional approaches are risky.

The aim of this study is to assess the modifications of squared coherence $\mathrm{K}^{2}$ between MAP and MCBFV variability in response to the induction of propofol general anesthesia in subjects undergoing coronary artery bypass graft (CABG) surgery. $\mathrm{K}^{2}$ was exploited to typify the $C A$ regime given that values of $\mathrm{K}^{2}$ close to 0 indicate a relative independence of MCBFV variations from MAP changes, while values close to 1 indicates perfect association between MAP and MCBFV variations. The null hypothesis of MAP-MCBF dynamical uncoupling is tested through the comparison of $\mathrm{K}^{2}$ values derived from the original paired MAP-MCBFV series with those derived from the random association of MAP series of one subject and MCBFV series taken from another one. 


\section{Methods}

\subsection{Experimental protocol}

Ten subjects (age 64.7+7.0 yrs, min-max: 52-75 yrs, 10 males) scheduled for elective CABG surgery were enrolled at the Department of Cardiothoracic, Vascular Anesthesia and Intensive Care, IRCCS Policlinico San Donato, Milan, Italy. The inclusion criteria were sinus rhythm, age over 18, absence of pathologies affecting brain or autonomic nervous system. The study was in keeping with Helsinki Declaration for studies involving human subjects and was approved by the ethical committee in charge at IRCCS Policlinico San Donato. Subjects signed an informed consent before participating. As described in $[9,10]$, subjects received atropine and fentanyl as premedication, then, intravenous anesthesia was induced and maintained through propofol (induction: bolus $1.5 \mathrm{mg} \cdot \mathrm{kg}^{-1}$; maintenance: $\left.3 \mathrm{mg} \cdot \mathrm{kg}^{-1} \cdot \mathrm{h}^{-1}\right)$ and remifentanil (maintenance: from 0.05 to $0.5 \mu \mathrm{g} \cdot \mathrm{kg}^{-1} \cdot \mathrm{min}^{-}$ ${ }^{1}$ ) infusion. During anesthesia subjects were mechanically ventilated at 12 to 16 breaths. min $^{-1}$ with $1: 1$ oxygen and air. Electrocardiogram (ECG), invasive AP from the radial artery and CBFV from the left middle cerebral artery, as derived with a transcranial Doppler device (Multi-Dop X, DWL, San Juan Capistrano, CA, USA) were simultaneously acquired through an A/D board connected to a laptop. Sampling rate was $1000 \mathrm{~Hz}$. Signals were recorded for six minutes before (PRE) and after (POST) the induction of anesthesia. Since out of the 10 enrolled subjects, 2 were acquired only during PRE, 6 during PRE and POST, and 2 only during POST, each group was composed by 8 subjects.

\subsection{Preprocessing procedures}

Heart period (HP), approximated as the time distance between two consecutive R-wave peaks on the ECG, was computed. MAP and MCBFV were derived as the integral between two consecutive diastolic points on the corresponding signal divided by the time interval between them [11]. The first diastolic point identifying the $\mathrm{i}^{\text {th }}$ MAP, or MCBFV, was taken inside the $i^{\text {th }}$ HP. All the measures were carried out on a beat-to-beat basis. Series were manually checked and ectopic beats or misdetections were corrected via linear interpolation. Corrections were less than $5 \%$ of the total series length. Synchronous series of 250 beats were picked up randomly in each experimental session for each subject. Mean and variance of HP, MAP and MCBFV series were computed, indicated as $\mu_{\mathrm{HP}}, \mu_{\mathrm{MAP}}, \mu_{\mathrm{MCBFV}}, \sigma_{\mathrm{HP}}^{2}, \sigma^{2} \mathrm{MAP}$ and $\sigma_{\mathrm{MCBFV}}^{2}$ and expressed respectively in $\mathrm{ms}, \mathrm{mmHg}, \mathrm{cm} \cdot \mathrm{s}^{-1}$, $\mathrm{ms}^{2}, \mathrm{mmHg}^{2}$ and $\mathrm{cm}^{2} \cdot \mathrm{s}^{-2}$.

\subsection{Spectral and cross-spectral analyses}

Parametric spectral analysis was carried out. MAP and MCBFV series were modeled as realizations of an autoregressive (AR) process. The AR coefficients were estimated via least squares method. The model order was optimized in the range from 10 to 14 via the Akaike information criterion. Power spectral density was factorized in components [12]. HP was used to scale the frequency axis of MAP and MCBFV power spectra and $\mathrm{K}^{2}$ function. Spectral components were labeled as very low frequency (VLF), low frequency (LF) or high frequency (HF) whether their central frequency was in the range $0.02-0.07,0.07-0.15$, and $0.15-0.4 \mathrm{~Hz}$ respectively. Compared to [1] the upper limit of LF band and lower limit of HF band was modified in relation to the ventilatory rate during POST. The absolute power of MAP and MCBFV series in the different frequency bands was computed, indicated as VLF $\mathrm{VHAP}_{\mathrm{M}} \mathrm{LF}_{\mathrm{MAP}}, \mathrm{HF}_{\mathrm{MAP}}$, VLF ${ }_{M C B F V}, \quad F_{M C B F V}$, and $\mathrm{HF}_{\mathrm{MCBFV}}$ and expressed respectively in $\mathrm{mmHg}^{2}$ and $\mathrm{cm}^{2} \cdot \mathrm{s}^{-2}$ in case of MAP and MCBFV series respectively. $\mathrm{K}^{2}$ function was assessed via parametric approach grounded on a bivariate AR model identification [13]. The coefficients were again estimated via least squares identification method and model order was fixed to 10 [13]. The average value of $\mathrm{K}^{2}$ in the three frequency bands was computed and indicated respectively as $\mathrm{K}^{2}{ }_{\mathrm{VLF}}, \mathrm{K}_{\mathrm{LF}}^{2}$ and $\mathrm{K}_{\mathrm{HF}}^{2}$. Values were bounded between 0 and 1 , where 0 indicated full MCBFV-MAP uncoupling and 1 perfect MCBFV-MAP coupling.

\subsection{Surrogate and statistical analyses}

The null hypothesis of uncoupling was tested by assessing $\mathrm{K}^{2}$ between the MAP series of one subject and the MCBFV series of another subject in the same experimental condition (i.e. PRE or POST). The null hypothesis of uncoupling was rejected whether $\mathrm{K}^{2}$ computed over the original pair was significantly different from that computed on surrogate pairs.

Unpaired t test, or Mann-Whitney rank sum test where appropriate, was applied to assess between-condition differences of time and frequency domain indices. Twoway analysis of variance (Holm-Sidak correction for multiple comparisons) was performed over $\mathrm{K}^{2}$ to evaluate the significance of the between-condition differences within the same type of analysis (i.e. over original or surrogate pairs) and between-analysis differences within the same experimental condition (i.e. PRE or POST). Results were reported as mean \pm standard deviation. A $p<0.05$ was always deemed as significant.

\section{Results}

Table 1 shows time domain indices derived from HP, 
Table 1. Time domain markers extracted from HP, MAP and MCBFV series during PRE and POST.

\begin{tabular}{ccc}
\hline Parameter & PRE & POST \\
\hline$\mu_{\mathrm{HP}}[\mathrm{ms}]$ & $915.63 \pm 219.14$ & $1101.23 \pm 112.27^{*}$ \\
$\sigma^{2} \mathrm{HP}\left[\mathrm{ms}{ }^{2}\right]$ & $758.40 \pm 923.93$ & $257.37 \pm 342.77$ \\
$\mu_{\mathrm{MAP}}[\mathrm{mmHg}]$ & $107.48 \pm 10.72$ & $71.33 \pm 11.20^{*}$ \\
$\sigma^{2} \mathrm{MAP}\left[\mathrm{mmHg}{ }^{2}\right]$ & $10.77 \pm 6.00$ & $2.03 \pm 1.15^{*}$ \\
$\mu_{\mathrm{MCBFV}}\left[\mathrm{cm}^{-1} \mathrm{~s}^{-1}\right]$ & $44.35 \pm 20.96$ & $38.66 \pm 16.60$ \\
$\sigma^{2} \mathrm{MCBFV}\left[\mathrm{cm}^{2} \cdot \mathrm{s}^{-2}\right]$ & $9.74 \pm 9.50$ & $5.08 \pm 4.22$ \\
\hline
\end{tabular}

$\mu_{\mathrm{HP}}=\mathrm{HP}$ mean; $\sigma^{2}{ }_{\mathrm{HP}}=\mathrm{HP}$ variance; $\mu_{\mathrm{MAP}}=\mathrm{MAP}$ mean; $\sigma^{2} \mathrm{MAP}=$ MAP variance; $\mu_{\mathrm{MCBFV}}=\mathrm{MCBFV}$ mean; $\sigma^{2} \mathrm{MCBFV}=$ MCBFV variance; $P R E=$ before general anesthesia induction; POST $=$ after general anesthesia induction. The symbol * indicates $p<0.05$ versus PRE.

MAP and MCBFV series during PRE and POST. During POST $\mu_{\mathrm{HP}}$ was increased, while $\mu_{\mathrm{MAP}}$ and $\sigma^{2}$ MAP were significantly reduced. MCBFV time domain indices were unchanged. Table 2 shows spectral markers derived from MAP and MCBV series in VLF, LF and HF bands. None of the spectral indices was significantly modified by anesthesia. The grouped error bar graphs in Fig.1 show $\mathrm{K}^{2}$ assessed in VLF (Fig.1a), LF (Fig.1b) and HF (Fig.1c) bands over original (black bars) and surrogate (white bars) pairs in PRE and POST. All the $\mathrm{K}^{2}$ indices assessed over original pairs during PRE were significantly higher than those computed over surrogate pairs. This result did not hold anymore during POST, because of the reduction of all the $\mathrm{K}^{2}$ markers of the original pairs in POST.

\section{Discussion}

The main findings of this study are: i) time domain indices identified the autonomic depression during POST; ii) $\mathrm{K}^{2}$ between MAP and MCBFV was significant during PRE and it was reduced during propofol general anesthesia; iii) irrespective of the frequency bands, MAP and MCBFV variabilities were uncoupled during POST.

Time domain indices reflected the expected propofolinduced autonomic depression [9]. Indeed, the significant decrease of $\sigma^{2}$ MAP and the evident reduction of $\sigma^{2} \mathrm{HP}$ suggest loss of sympathetic tone and vagal withdrawal. Modifications of MAP and MCBFV spectral markers during POST were less evident, likely due to the small size of the group. Regardless of the frequency band, $\mathrm{K}^{2}$ was significantly above the level set by surrogate analysis during PRE. Since a perfectly working CA would lead to a full MCBFV-MAP decoupling, our data suggest that during PRE this regime was not fully attained. Remarkably, our values of $\mathrm{K}^{2}$ found during PRE (i.e. $0.38,0.29,0.27$ respectively in VLF, LF and HF bands) are in agreement with those reported in literature [1]. Closed loop relationship between MAP and MCBFV series, resulting from the pressure-flow dependence and
Table 2. Frequency domain markers extracted from MAP and MCBFV series during PRE and POST.

\begin{tabular}{ccc}
\hline Parameter & PRE & POST \\
\hline $\mathrm{VLF}_{\mathrm{MAP}}\left[\mathrm{mmHg}^{2}\right]$ & $6.08 \pm 7.10$ & $0.06 \pm 0.07$ \\
$\mathrm{LF}_{\mathrm{MAP}}\left[\mathrm{mmHg}^{2}\right]$ & $0.39 \pm 0.47$ & $0.02 \pm 0.01$ \\
$\mathrm{HF}_{\mathrm{MAP}}\left[\mathrm{mmHg}^{2}\right]$ & $2.40 \pm 4.21$ & $1.70 \pm 1.18$ \\
$\mathrm{VLF}$ & $5.49 \pm 9.88$ & $0.55 \pm 0.87$ \\
$\mathrm{LF}_{\mathrm{MCBFV}}\left[\mathrm{cm}^{2} \cdot \mathrm{cm}^{-2}\right]$ & $0.61 \pm 0.95$ & $1.19 \pm 1.78$ \\
$\mathrm{HF}_{\mathrm{MCBFV}}\left[\mathrm{cm}^{2} \cdot \mathrm{s}^{-2}\right]$ & $0.68 \pm 0.80$ & $1.31 \pm 1.16$ \\
\hline
\end{tabular}

$\mathrm{VLF}=$ very low frequency; $\mathrm{LF}=$ low frequency; $\mathrm{HF}=$ high frequency; $\mathrm{PRE}=$ before general anesthesia induction; POST $=$ after general anesthesia induction. The symbol * indicates $p<0.05$ versus PRE.

the Cushing reflex and evoking a MAP increase in response to hypoperfusion, would lead to a significant $\mathrm{K}^{2}$ $[3,14]$. However, the measured amount of $\mathrm{K}^{2}$, although sufficient to reject the null hypothesis of MCBFV-MAP uncoupling based on our surrogate test, is quite limited, thus suggesting that the MCBFV-MAP coupling is weak on average and likely to be interrupted by periods of full uncoupling. These small values are compatible with an active and working CA operating to achieve dynamically the condition of MCBFV-MAP independence. During POST $\mathrm{K}^{2}$ was significantly reduced, hence the null hypothesis of MCBFV-MAP uncoupling could not be rejected anymore. This result is in disagreement with [8] showing much higher values of $\mathrm{K}^{2}$ during propofol anesthesia. However, in [8] the level of sedation was lighter and the population was younger and healthy. Since CA should aim at decoupling MCBFV from MAP variability, our results support the notion of preservation of CA during propofol anesthesia reported in [8]. Anyway, the observed level of MCBFV-MAP uncoupling during POST indicate that the dynamical condition of low, but significant, level of association between MCBFV and MAP fluctuations observed during PRE is lost. This absent level of MCBFV-MAP coupling during POST might be a hallmark of no sufficient challenge (i.e. MAP variations are reduced to a negligible level during POST). Therefore, this negligible level of $\mathrm{K}^{2}$ cannot be robustly taken as a full preservation of the CA and advocates the need of an intervention modifying MAP to verify the ability of CA to keep MCBFV stable. When an intervention was applied to modify AP, via thigh cuff rapid deflation or administration of vasoactive drug during propofol anesthesia at doses similar to those of the present study, the CA was found to be preserved [7]. However, the strategy adopted in this study to compute the final $\mathrm{K}^{2}$ marker from the $\mathrm{K}^{2}$ function (i.e. the average in the assigned band) could hide significant peak values, especially in HF band. We thus advocate additional studies given the relevance of preserving CA during propofol general anesthesia. Remarkably, the most relevant implication of our findings is that transfer 

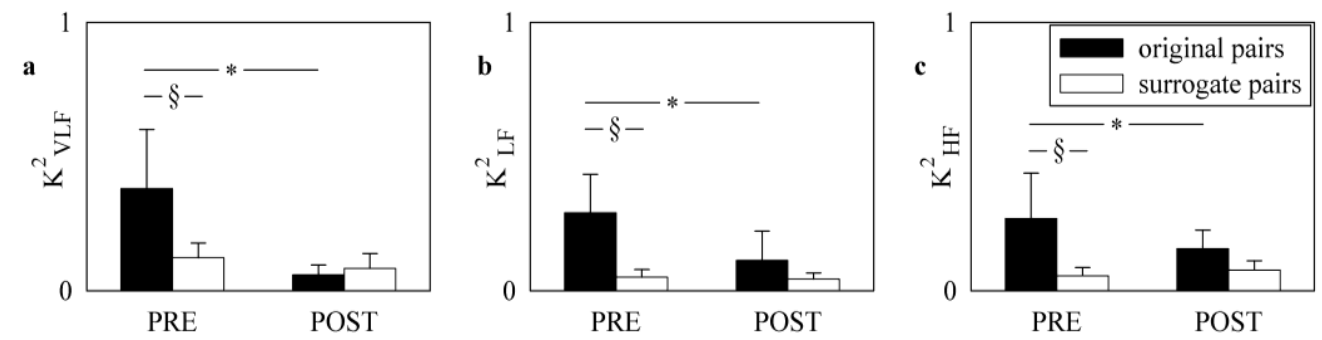

Figure 1. Grouped error bar graphs show $\mathrm{K}^{2}$ assessed between MAP and MCBFV series in VLF (a), LF (b) and HF (c) bands over original (black bars) and surrogate (white bars) pairs during PRE and POST. The symbols * and § indicate a significant difference versus PRE within the same type of analysis and versus original pairs within the same experimental condition with $p<0.05$.

function-based approach, frequency exploited in CA assessment [1], cannot be exploited during POST because a significant amount of MCBFV-MAP correlation is a prerequisite for the robust estimation of transfer function.

\section{Conclusions}

This study investigates CA modifications in response to propofol general anesthesia induction in subjects undergoing $\mathrm{CABG}$ surgery via coherence analysis of spontaneous MAP and MCBFV fluctuations. The null hypothesis of MCBFV-MAP uncoupling was rejected during PRE, while during POST the MCBFV-MAP association was weakened to a level that the null hypothesis of MCBFV-MAP uncoupling could not be rejected. Whether confirmed on a larger database, our findings suggest that the observed reduction of the coupling between MAP and MCBFV during POST is in keeping with the aim of CA, even though a full decoupling during propofol general anesthesia might hide the inability of the cerebrovascular regulatory system to cope with potentially risky situations as hypertension and hypoperfusion.

\section{Acknowledgments}

The study was supported by Italian Ministry of Health Grant GR-2013-02356272.

\section{References}

[1] J. A. Claassen, A. S. Meel-van den Abeelen, D. M. Simpson, R. B. Panerai and international Cerebral Autoregulation Research Network (CARNet), "Transfer function analysis of dynamic cerebral autoregulation: A white paper from the International Cerebral Autoregulation Research Network," J. Cereb. Blood Flow Metab., vol. 36, pp. 665-680, 2016.

[2] R. Aaslid, K. F. Lindegaard, W. Sorteberg and H. Nornes "Cerebral autoregulation dynamics in humans," Stroke, vol. 20, pp. 45-52, 1989.

[3] V. Bari et al., "Cerebrovascular and cardiovascular variability interactions investigated through conditional joint transfer entropy in subjects prone to postural syncope,"
Physiol. Meas., vol. 38, pp. 976-991, 2017.

[4] J. W. Hamner, C. O. Tan, K. Lee, M. A. Cohen and J. A. Taylor, "Sympathetic control of the cerebral vasculature in humans," Stroke, vol. 41, pp. 102-109, 2010.

[5] S. Strandgaard and O. B. Paulson, "Regulation of cerebral blood flow in health and disease," J. Cardiovasc. Pharmacol., vol. 19, pp. S89-S93, 1992.

[6] E. Vaini et al., "Causality analysis reveals the link between cerebrovascular control and acute kidney dysfunction after coronary artery bypass grafting," Physiol Meas., vol. 40, pp. 064006, 2019.

[7] S. Strebel et al., "Dynamic and static cerebral autoregulation during isoflurane, desflurane, and propofol anesthesia," Anesthesiology, vol. 83, pp. 66-76, 1995.

[8] Y. Ogawa et al., "The different effects of midazolam and propofol sedation on dynamic cerebral autoregulation," Anesth. Analg., vol. 111, pp. 1279-1284, 2010.

[9] A. Porta et al., "Model-based causal closed-loop approach to the estimate of baroreflex sensitivity during propofol anesthesia in patients undergoing coronary artery bypass graft," J. Appl. Physiol., vol. 115, pp. 1032-1042, 2013.

[10] M. Ranucci, A. Porta, V. Bari, V. Pistuddi and M. T. La Rovere, "Baroreflex sensitivity and outcomes following coronary surgery," PLoS One, vol. 12, pp. e0175008, 2017.

[11] V. Bari et al., "Nonlinear effects of respiration on the crosstalk between cardiovascular and cerebrovascular control systems," Phil. Trans. R. Soc. A, vol. 374, 20150179, 2016.

[12] G. Baselli, A. Porta, O. Rimoldi, M. Pagani and S. Cerutti, "Spectral decomposition in multichannel recordings based on multivariate parametric identification," IEEE Trans. Biomed. Eng., vol. 44, pp. 1092-1101, 1997.

[13] A. Porta, G. Baselli, O. Rimoldi, A. Malliani and M. Pagani, "Assessing baroreflex gain from spontaneous variability in conscious dogs: role of causality and respiration," Am. $J$. Physiol., vol. 279, pp. H2558-H2567, 2000.

[14] S Saleem et al., "Is the Cushing mechanism a dynamic blood pressure-stabilizing system? Insights from Granger causality analysis of spontaneous blood pressure and cerebral blood flow," Am. J. Physiol. vol. 315, pp. R484R495.

Address for correspondence:

Dr Vlasta Bari, PhD

IRCCS Policlinico San Donato

Via F.Fellini 4, 20097 San Donato Milanese, Milan, Italy

E-mail: vlasta.bari@grupposandonato.it 